\title{
Predicting Sales of Cross-chain Discrete Manufacturing Products Based on LSTM
}

\author{
Zhicheng Wang ${ }^{1}$, Ye Tao ${ }^{2}$, Xiangqian Ding ${ }^{1}$, Ruichun Hou ${ }^{1}$, Hui Zhang ${ }^{1}$ \\ \{wangzhicheng@stu.ouc.edu.cn, ye.tao@qust.edu.cn , \\ hrch4968@sina.com $\}$ \\ ${ }^{1}$ College of Information Science and Engineering, Ocean University of China, Qingdao, Shandong, \\ China, \\ ${ }^{2}$ School of Information Science and Technology, Qingdao University of Science and Technology, \\ Qingdao, China
}

\begin{abstract}
In the mass customization product value chain, the sales forecast in the marketing chain is important. Traditional forecasting methods only consider the previous sales volume, which is a single value chain factor. In fact, with the rapid development of online media, the factors affecting the sales of manufacturing products have become increasingly complex. For example, the attention and evaluation of online social media is an important influence on sales forecasts. In addition, other influencing factors such as stocks also have a certain impact on product sales. Therefore, it is possible to integrate cross-domain information to improve the accuracy of prediction results. In this paper, we propose an LSTM model with the portal's attention index, and involve factors from multiple domains, such as the network media, market environment and original product sales volume. Experiment results show that using the proposed cross-domain approach obtains more accurate prediction results than other mainstream models.
\end{abstract}

Keywords: value chain, cross-chain prediction, LSTM.

\section{Introduction}

In modern mass customization manufacturing, product sales forecasting is one of the important issues in business intelligence. Accurately predicting product sales trends can help companies better grasp market demand, adjust output and optimize inventory. In the modern market, the product life cycle has become a "pencil type" with extremely short-lived peaks. It is no longer a parabola-like slow change, but straight up and down. Product life cycles are getting shorter and shorter, so sales forecasting is becoming more important. This makes the senior management of the enterprise must formulate flexible marketing and inventory allocation strategies for the rapidly changing market, and the forecast of sales volume will be an important reference for the senior management of the enterprise to formulate corporate strategies.

Most forecasting methods are built upon time series models with single input and have been applied in some specific actual scenarios and has a good forecasting effect. Traditional time series forecasting methods fit historical time trend curves by establishing appropriate mathematical models, but the data they rely on are simple and often face lag problems. 
With the continuous improvement of the Industrial Internet system, it is possible to obtain multidimensional data from multiple value chains. For example, with the development of the Internet, the attention of various brands on the Internet has had a significant impact on sales. Many consumers can post their own evaluation of a certain product on social media, and the impact of consumer evaluation on sales is becoming more and more important. Therefore, many scholars [1]- [3] find that using cross-domain data to predict sales can get better results through in-depth research.

This article conducts an experiment on the time series of sales volume of a manufacturing industry, and collects four types of cross-value chain data: sales data, user evaluation, Internet attention index, and corporate stock price fluctuations of 18 different manufacturers. According to the actual situation, the data are optimized for the influence of de-seasonal factors. Different from the data of a single sales value chain used in previous studies, this article uses four types of cross-value chain data to predict the sales volume of a manufacturing industry through the LSTM model. The experiment compares the impact of cross-value chain data and single value chain data on the accuracy of sales volume prediction, the effect of de-seasonal data and seasonality data on the accuracy of sales volume prediction, and the impact of using LSTM model and LSSVR model on sales volume prediction. The experimental results show that compared with the past, the experimental results have been greatly improved.

The rest of this study is organized as follows. Related work about predicting sales is introduced in Section 2. Section 3 presents methodologies utilized in this study and the proposed framework. Section 4 depicts the results of this study. Conclusions are delivered in Section 5.

\section{Related work}

There are many traditional prediction methods for time series data. F. M. De and X. Yao [4], [5] used the traditional method to forecast the load of the power grid, and used the sales volume of the previous day as the input to forecast the sales volume data of the next day, and achieved a certain forecast effect. However, only the data of previous day is used, and the prediction result of the previous data input as input for the time series model is more error. D. Trigg and A. Leach [6], [7]used the exponential smoothing method to predict the discrete signals and achieved a certain prediction effect. Exponential smoothing is based on previous predictions and the percentage of prediction errors. Because of the simplicity, transparency, and ability to capture various time series data patterns. In recent years, exponential smoothing has become one of the most popular and practical methods in time series prediction. However, for the sales forecast of manufacturing products, it is clear that the index smoothing model cannot accurately reflect the market and predict sales volume. Therefore, the method of index smoothing is not applicable to the sales forecast of manufacturing products in modern volatile market.

In recent years, with the development of artificial intelligence algorithms[8], BPNN [9] and SVR [10] algorithms have also become common methods for predicting time series data. In previous related studies, some scholars used the BPNN [11], [12] algorithm to predict time series data. In some areas, such as automobile sales forecasting, certain results have been achieved. But for the BPNN algorithm, it requires a large amount of data, and the prediction result of small samples is not ideal. For small sample predictions, the SVR [13], [14]algorithm performs better. Therefore, in recent years, many scholars have used SVR algorithms to predict time series data and have achieved some results. However, the SVR algorithm has poor practicability and poor generalization ability. 
In recent years, with the development of online social media, the value chain of online social media has become an important factor affecting sales. Previously, some scholars have also done a lot of related work to prove that the promotion and evaluation of social media can affect the sales of manufactured products[15]-[17]. At the same time, some scholars have also pointed out that market macro performance, such as stock information, industrial indexes and other indicators, will also affect the sales of manufacturing products[18], [19].

In these studies, LIU and PAI [20]used the LSSVR model to model car sales, taking past car sales, Twitter reviews, and the Dow Jones Industrial Index as inputs, and predicting car sales for the next month through training. However, in this article, the Dow Jones Industrial Index as a macro value that represents the overall market does not reflect the performance of different companies and manufacturers in the capital market. Therefore, there is still room for improvement in the forecast results. This article will focus on comparing the LSTM prediction model with LIU's LSSVR model, emphasizing that the prediction accuracy of prediction models across multiple value chains is greatly improved compared with the prediction results of single value chain prediction models.

\section{Predicting based method}

\subsection{Basic framework}

We use two types of data sets for prediction. The first type is the total monthly sales of home appliances from different manufacturers, and the second type is independent variable data, which includes Weibo users' sentiment on the corresponding manufacturers' products, and the Baidu index of the corresponding manufacturers' products. And the stock price of the corresponding manufacturers.
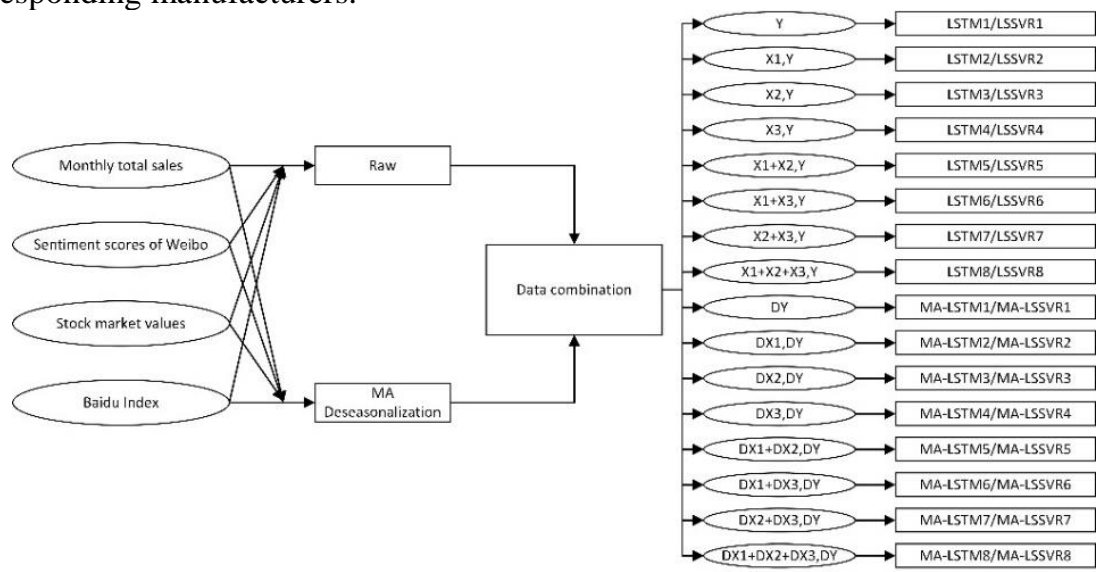

Fig. 1. The proposed monthly total sales predicting framework

We performed experiments in two groups, one using raw data and the other using moving average (MA) to remove seasonal factors. The data across multiple chains are then used as input to be trained in the LSTM model and the LSSVR model, and the prediction results are obtained. The algorithm flow chart in this paper is shown in Fig. 1. 


\subsection{Deasonalization method}

If the value of the time series at time $t$ can be explained by a linear combination of time $t$ and $i$ error terms before time $t$, then a moving average MA(q) model can be used to predict the value at time $t$.

$$
y_{t}=\varepsilon_{t}+\sum_{i=1}^{q} \beta_{i} \varepsilon_{t-i}+\mu
$$

In Eq.(2): $y_{t}$ represents the value of the predicted time series at time $t ; \varepsilon_{\mathrm{t}}$ and $\varepsilon_{t-i}$ represent the random errors at time $t$ and $t-i$ respectively; $q$ represents the order of the model; $\beta_{i}$ is the regression parameter; $\mu$ represents a constant term. In the following sections, we will use the MA algorithm to remove the seasonal factor operation.

In the following sections, we will use the MA algorithm to remove the seasonal factor operation.

\subsection{LSTM (Long Short-Term Memory) method}

In 1997, Hochreiter first proposed Long Short-Term Memory (LSTM), which is a special recurrent neural network (RNN), which can effectively solve the problem of gradient disappearance or gradient explosion of RNN, and can learn long-term dependencies. Compared with RNN, LSTM is designed for the controller of the neural cell, which can judge whether the information is useful. The Cell control unit is shown in the figure.

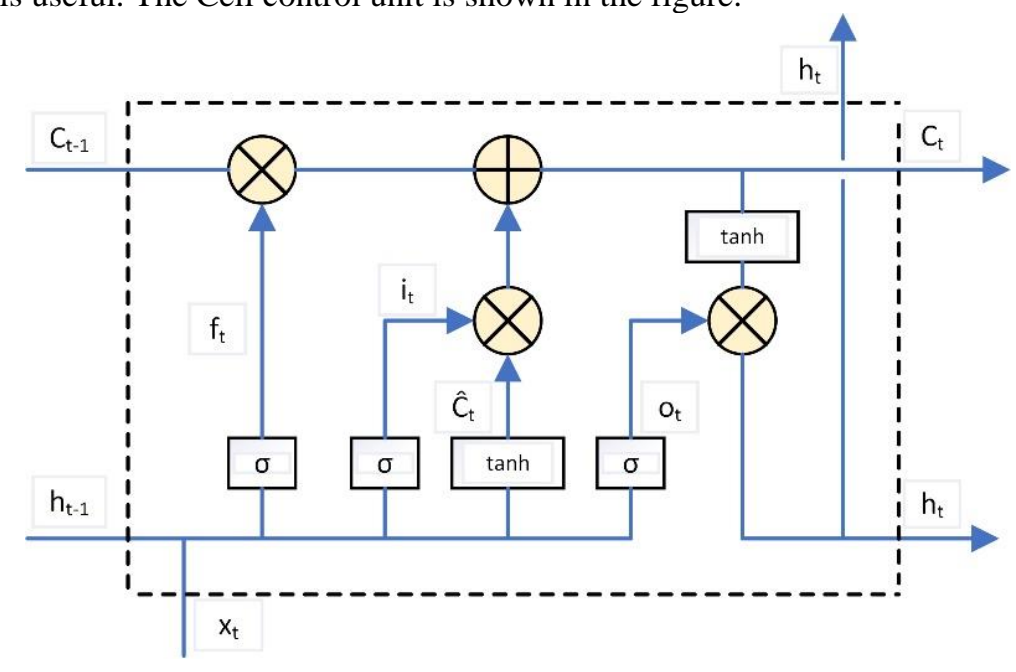

Fig. 2. Cell structure in LSTM model

The control unit in the LSTM model consists of a memory unit $C$ for recording the current state and three gates that control the flow of data. These three gates are composed of input gate 
$i$, output gate $o$, and forget gate $f$. At time $t$, after the data enters the control unit, through calculation, the LSTM can choose to remember or forget some information, output the control information, and pass these status information to the next time $t+1$.

The forget gate is the information that determines what we will discard from the state of the cell. The gate reads $h_{t-1}$ and $x_{t}$ and outputs a value in the range $0-1$ to the number in state $C_{t}$ of the previous output. 1 means "completely reserved", 0 means "completely discarded". The calculation method is shown as Eq.(3). Where $f_{t}$ represents the forget gate information at time $t$.

$$
f_{t}=\sigma \cdot\left(w_{f} \cdot\left[h_{t-1}, x_{t}\right]+b_{f}\right)
$$

The input gate determines how much new information is added to the cell state. The calculation method is shown as Eqs.(4-5). Among them, $i_{t}$ indicates the input gate information at time $t$, and $C_{t}$ indicates the update of the memory unit, which indicates how much information is forgotten and which of the current input information needs to be updated into the current memory unit.

$$
\begin{array}{r}
i_{t}=\sigma \cdot\left(w_{t} \cdot\left[h_{t-1}, x_{t}\right]+b_{i}\right) \\
C_{t}=f_{t} \cdot c_{t-1}+i_{t} \cdot \tanh \left(w_{c}-\left[h_{t-1}, x_{t}\right]+b_{c}\right)
\end{array}
$$

The output gate determines what value to output. The calculation method is shown as Eqs.(6-7). Among them, $o_{t}$ indicates that the door information is output at time $t$, which is used to control the update of the information to achieve the purpose of increasing and decreasing the information. $h_{t}$ produces the current output result, and the output gate decides which information is finally output.

$$
\begin{gathered}
o_{t}=\sigma \cdot\left(w_{o} \cdot\left[h_{t-1}, x_{t}\right]+b_{o}\right) \\
h_{t}=o_{t} \cdot \tanh \left(C_{t}\right)
\end{gathered}
$$

In the above formula, $\sigma$ represents the sigmoid function, and $w_{f}, w_{i}, w_{o}, b_{f}, b_{i}$ and $b_{o}$ represent the weights and offset values of the three gates, respectively.

\subsection{LSSVR (least square support vector regression) method}

The least-square support vector regression (LSSVR) model is one of the modified techniques which can reduce the computation load of the original support vector regression model. Suykens et al. Proposed the least squares support vector regression (LSSVR) model based on the standard SVR. LSSVR changed the inequality constraints of the SVR to equality constraints, which made the solution of the original convex quadratic programming problem into a solution of linear equations, which greatly reduced the calculation difficulty and calculation time.

Given a set of training samples $\left\{x_{i}, y_{i} \mid i=1,2, \ldots, M\right\}, x_{i} \in R^{P}$ and $y_{i} \in R$ represent the input and expected output of the model, respectively, and $M$ represents the number of samples. $f(x)$ represents the mapping relationship between model input $\mathrm{x}$ and output $\mathrm{y}$. The calculation formula is as follows: 


$$
f(x)=x^{T} w+b
$$

In Eq.(8), w and b are weight vectors and offset terms, respectively. In order to solve for the appropriate $w$ and $b$, the loss function of LSSVR needs to be calculated:

$$
\begin{aligned}
\min : \frac{1}{2}\|w\|^{2}+\frac{1}{2} y \sum_{i=1}^{M} e_{i}^{2} \\
\text { s.t. } x_{i}^{T} w+b-y_{i}=e_{i}
\end{aligned}
$$

Finally, the equation is solved. The prediction function $f(x)$ of LSSVR is expressed as:

$$
f(x)=\sum_{i=1}^{M} \alpha_{i} x^{T} x_{i}+b
$$

\section{Experiments and discussions}

\subsection{Data set description}

We use two types of data sets for prediction. The first type is the total monthly sales of home appliances from different manufacturers, and the second type is independent variable data, which includes Weibo users' sentiment on the corresponding manufacturers' products, and the Baidu index of the corresponding manufacturers' products. And the stock price of the corresponding manufacturers.

The data of manufacturers' home appliances from 2012.1-2018.6 was provided by related companies. There are a total of 18 different product models. Related data involves confidentiality. The product data of Weibo users from 2012.1-2017.12 was found out by the crawler code, and the Weibo were collected by keywords, that is, "buyer electricity". Through text analysis, distinguish and count whether the user's evaluation of the product is positive or negative.2012.1-2017.12 Baidu index data is the number of times the manufacturer was searched and followed in the Baidu search engine. The stock data of 2012.1-1017.12 is the percentage change of stocks of corresponding manufacturers for the month.

\subsection{Data preprocessing and model building}

In this study, a time series model and a multivariate regression model were performed to predict total monthly sales of white goods. This article will predict the overall sales of white goods for two-time series models, LSSVR and LSTM. In addition, the non-seasonal deseasonalization procedure obtained from the monthly white goods sales data applies to the monthly white goods sales data, popularity scores, Baidu index, and stock market value. The three independent variable data are arranged and combined, and 16 LSTM / LSSVR models are listed to use different data combinations to predict the total monthly sales volume and study it. 
Table 1. Data types and corresponding codes

\begin{tabular}{llll}
\hline LSSVR/RNN models & Data used & LSSVR/RNN models & Data used \\
\hline LSTM/LSSVR 1 & Y & MA-LSTM/MA-LSSVR 1 & DY \\
LSTM/LSSVR 2 & X1, Y & MA-LSTM/MA-LSSVR 2 & DX1, DY \\
LSTM/LSSVR 3 & X2, Y & MA-LSTM/MA-LSSVR 3 & DX2, DY \\
LSTM/LSSVR 4 & X3, Y & MA-LSTM/MA-LSSVR 4 & DX3, DY \\
LSTM/LSSVR 5 & (X1, X2), Y & MA-LSTM/MA-LSSVR 5 & (DX1, DX2), DY \\
LSTM/LSSVR 6 & (X1, X3), Y & MA-LSTM/MA-LSSVR 6 & (DX1, DX3), DY \\
LSTM/LSSVR 7 & $(\mathrm{X} 2, \mathrm{X} 3), \mathrm{Y}$ & MA-LSTM/MA-LSSVR 7 & (DX2, DX3), DY \\
LSTM/LSSVR 8 & $(\mathrm{X} 1, \mathrm{X} 2, \mathrm{X} 3), \mathrm{Y}$ & MA-LSTM/MA-LSSVR 8 & (DX1, DX2, DX3), \\
& & & DY \\
\hline
\end{tabular}

Table 2. LSSVRILSTM models and data used for predicating monthly total sales

\begin{tabular}{ll}
\hline Data codes & Data descriptions \\
\hline X1 & Sentiment scores of Weibo \\
X2 & Baidu index \\
X3 & Percent change in stocks Market values \\
DX1 & Deseasonalized sentiment scores of Weibo \\
DX2 & Deseasonalized Baidu index \\
DX3 & Deseasonalized Percent change in stocks Market values \\
Y & Monthly total sales \\
DY & Deseasonalized Monthly total sales \\
\hline
\end{tabular}

In the flowchart, we divide the independent variables into two cases, one is the original data, and the other is fed into the MA seasonal factor algorithm, and then the independent variables and sales are combined and fed into the LSTM / LSSVR model for training.

\subsection{The results}

In this section, the results of predicting monthly total sales by time series models and multivariate regression models with various data types are illustrated. The predicting performance is measured by MAPE, WAPE[21] and NMAE[22] are shown as Eqs. (12-14).

$$
\begin{aligned}
& \operatorname{MAPE}(\%)=\frac{100}{N} \sum_{t=1}^{N}\left|\frac{Y_{t}-F_{t}}{Y_{t}}\right| \\
& W A P E(\%)=100 \frac{\sum_{t=1}^{N}\left|F_{t}-Y_{t}\right|}{\sum_{t=1}^{N} Y_{t}} \\
& N M A E=\frac{1}{Y_{h}-Y_{l}}\left[\frac{1}{N} \sum_{t=1}^{N}\left|Y_{t}-F_{t}\right|\right]
\end{aligned}
$$

where $N$ is the number of predicting periods, $Y_{t}$ is the actual value at period $t$, and $F_{t}$ is the forecasting value at period $t, Y_{h}$ is highest actual value, and $Y_{l}$ is the lowest actual value. 


\section{Comparison of results using seasonal data}

Table 3. MAPE, WAPE and NMAE values by using LSTM/LSSVR models with seasonal data of independent variables and original monthly total sales to predict monthly total sales.

\begin{tabular}{|c|c|c|c|c|c|c|c|}
\hline \multirow{3}{*}{$\begin{array}{l}\text { LSTM } \\
\text { Models }\end{array}$} & MAPE & \multirow{3}{*}{$\begin{array}{l}\text { LSSVR } \\
\text { Models }\end{array}$} & MAPE & \multirow{3}{*}{$\begin{array}{l}\text { LSTM } \\
\text { Models }\end{array}$} & MAPE & \multirow{3}{*}{$\begin{array}{l}\text { LSSVR } \\
\text { Models }\end{array}$} & MAPE \\
\hline & WAPE & & WAPE & & WAPE & & WAPE \\
\hline & NMAE & & NMAE & & NMAE & & NMAE \\
\hline \multirow{3}{*}{ LSTM1 } & $12.36 \%$ & \multirow{3}{*}{ LSSVR1 } & $24.10 \%$ & \multirow{3}{*}{ LSTM5 } & $11.08 \%$ & \multirow{3}{*}{ LSSVR5 } & $20.72 \%$ \\
\hline & $9.83 \%$ & & $16.59 \%$ & & $7.43 \%$ & & $14.86 \%$ \\
\hline & 0.030 & & 0.050 & & 0.024 & & 0.044 \\
\hline \multirow{3}{*}{ LSTM2 } & $12.23 \%$ & \multirow{3}{*}{ LSSVR2 } & $21.18 \%$ & \multirow{3}{*}{ LSTM6 } & $7.78 \%$ & \multirow{3}{*}{ LSSVR6 } & $21.60 \%$ \\
\hline & $8.43 \%$ & & $15.12 \%$ & & $5.71 \%$ & & $15.61 \%$ \\
\hline & 0.046 & & 0.046 & & 0.018 & & 0.047 \\
\hline \multirow{3}{*}{ LSTM3 } & $11.83 \%$ & \multirow{3}{*}{ LSSVR3 } & $21.51 \%$ & \multirow{3}{*}{ LSTM7 } & $8.42 \%$ & \multirow{3}{*}{ LSSVR7 } & $21.79 \%$ \\
\hline & $8.28 \%$ & & $15.64 \%$ & & $6.16 \%$ & & $15.71 \%$ \\
\hline & 0.026 & & 0.047 & & 0.019 & & 0.047 \\
\hline \multirow{3}{*}{ LSTM4 } & $9.38 \%$ & \multirow{3}{*}{ LSSVR4 } & $23.64 \%$ & \multirow{3}{*}{ LSTM8 } & $7.51 \%$ & \multirow{3}{*}{ LSSVR8 } & $21.05 \%$ \\
\hline & $6.32 \%$ & & $16.56 \%$ & & $5.51 \%$ & & $15.00 \%$ \\
\hline & 0.019 & & 0.050 & & 0.017 & & 0.045 \\
\hline
\end{tabular}

It can be seen from Table 3 that when using data without seasonal factors for prediction, when the number of independent data combinations increases, the values of WAPE and NMAE decrease significantly. It can be seen that for the sales forecast of this type of product, the more independent variables, the smaller the error percentage of the predicted sales. For the three independent variables of Weibo's product evaluation, the Baidu index, and corporate stock percentage change, the most relevant to the sales forecast is the stock percentage change.

As can be seen from Table 3, comparing the LSTM model with the LSSVR model, the values of MAPE and NMAE predicted by the LSTM model are significantly smaller, indicating that the error percentage of the LSTM model predicted sales is smaller. At the same time, the NMAE value predicted by the LSTM model is also smaller, indicating that the LSTM is more accurate in predicting extreme values in the prediction. It can be seen that for this type of product sales forecast, the prediction effect of the LSTM model is significantly better than the prediction effect of the LSSVR model.

In this paper, five types of data, real sales, LSTM1, LSSVR1, LSTM8, and LSSVR8, are selected for comparison.as shown in Fig. 3. 


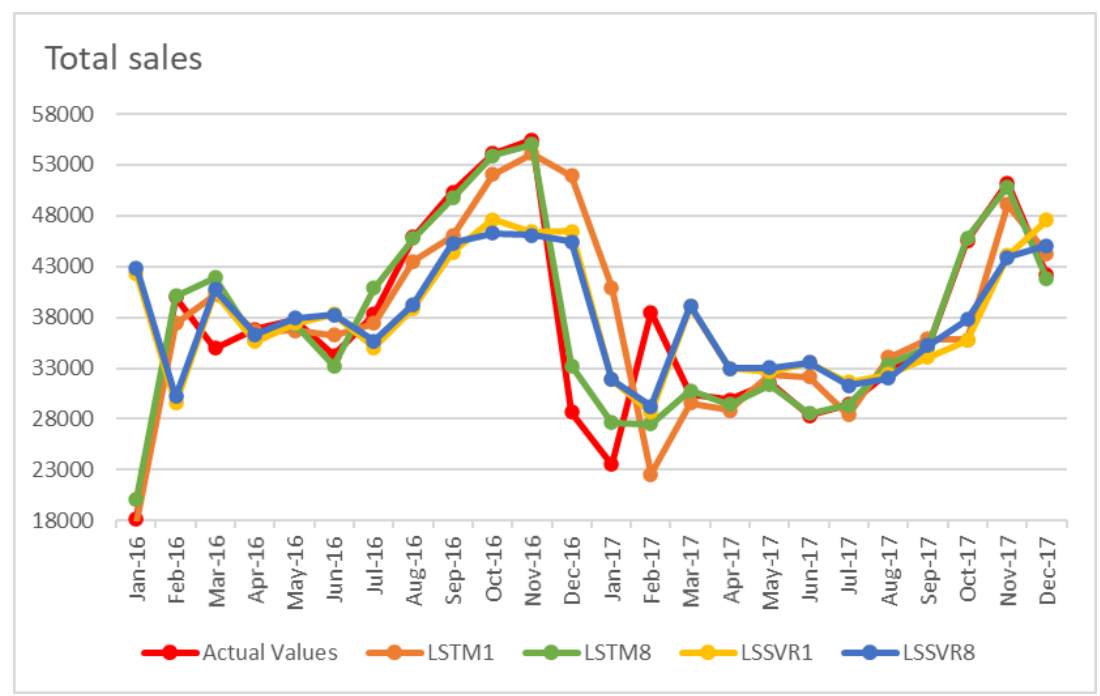

Fig. 3. Actual and predicted monthly total sales of LSTM/LSSVR models.

\section{Comparison of results using deseasonal data}

Table 4. MAPE, WAPE and NMAE values by using MA-LSTM/MA-LSSVR models with deseasona data of independent variables and original monthly total sales to predict monthly total sales.

\begin{tabular}{|c|c|c|c|c|c|c|c|}
\hline \multirow{3}{*}{$\begin{array}{l}\text { LSTM } \\
\text { Models }\end{array}$} & MAPE & \multirow{3}{*}{$\begin{array}{l}\text { LSSVR } \\
\text { Models }\end{array}$} & MAPE & \multirow{3}{*}{$\begin{array}{l}\text { LSTM } \\
\text { Models }\end{array}$} & MAPE & \multirow{3}{*}{$\begin{array}{l}\text { LSSVR } \\
\text { Models }\end{array}$} & MAPE \\
\hline & WAPE & & WAPE & & WAPE & & WAPE \\
\hline & NMAE & & NMAE & & NMAE & & NMAE \\
\hline \multirow{3}{*}{$\begin{array}{c}\text { MA- } \\
\text { LSTM1 }\end{array}$} & $12.44 \%$ & \multirow{3}{*}{$\begin{array}{c}\text { MA- } \\
\text { LSSVR1 }\end{array}$} & $16.04 \%$ & \multirow{3}{*}{$\begin{array}{l}\text { MA- } \\
\text { LSTM5 }\end{array}$} & $8.71 \%$ & \multirow{3}{*}{$\begin{array}{c}\text { MA- } \\
\text { LSSVR5 }\end{array}$} & $15.27 \%$ \\
\hline & $9.41 \%$ & & $11.55 \%$ & & $6.44 \%$ & & $10.92 \%$ \\
\hline & 0.028 & & 0.034 & & 0.019 & & 0.032 \\
\hline \multirow{3}{*}{$\begin{array}{c}\text { MA- } \\
\text { LSTM2 }\end{array}$} & $10.65 \%$ & \multirow{3}{*}{$\begin{array}{c}\text { MA- } \\
\text { LSSVR2 }\end{array}$} & $15.75 \%$ & \multirow{3}{*}{$\begin{array}{c}\text { MA- } \\
\text { LSTM6 }\end{array}$} & $6.76 \%$ & \multirow{3}{*}{$\begin{array}{c}\text { MA- } \\
\text { LSSVR6 }\end{array}$} & $15.67 \%$ \\
\hline & $7.42 \%$ & & $11.29 \%$ & & $4.40 \%$ & & $11.32 \%$ \\
\hline & 0.022 & & 0.034 & & 0.013 & & 0.034 \\
\hline \multirow{3}{*}{$\begin{array}{c}\text { MA- } \\
\text { LSTM3 }\end{array}$} & $9.27 \%$ & \multirow{3}{*}{$\begin{array}{c}\text { MA- } \\
\text { LSSVR3 }\end{array}$} & $15.13 \%$ & \multirow{3}{*}{$\begin{array}{c}\text { MA- } \\
\text { LSTM7 }\end{array}$} & $6.94 \%$ & \multirow{3}{*}{$\begin{array}{c}\text { MA- } \\
\text { LSSVR7 }\end{array}$} & $15.23 \%$ \\
\hline & $7.04 \%$ & & $11.19 \%$ & & $4.62 \%$ & & $11.24 \%$ \\
\hline & 0.021 & & 0.033 & & 0.014 & & 0.033 \\
\hline \multirow{3}{*}{$\begin{array}{c}\text { MA- } \\
\text { LSTM4 }\end{array}$} & $7.18 \%$ & \multirow{3}{*}{$\begin{array}{c}\text { MA- } \\
\text { LSSVR4 }\end{array}$} & $15.80 \%$ & \multirow{3}{*}{$\begin{array}{c}\text { MA- } \\
\text { LSTM8 }\end{array}$} & $6.85 \%$ & \multirow{3}{*}{$\begin{array}{c}\text { MA- } \\
\text { LSSVR8 }\end{array}$} & $15.36 \%$ \\
\hline & $4.63 \%$ & & $11.47 \%$ & & $4.22 \%$ & & $10.95 \%$ \\
\hline & 0.014 & & 0.034 & & 0.013 & & 0.032 \\
\hline
\end{tabular}

As can be seen from Table 4, when using the data without seasonal factors for prediction, as the number of independent data combinations increases, the values of WAPE and NMAE decrease significantly. It can be seen that for the sales forecast of this type of product, the more independent variables, the smaller the error percentage of the predicted sales. For the three 
independent variables of Weibo's product evaluation, the Baidu index, and corporate stock percentage change, the most relevant to the sales forecast is the stock percentage change.

Comparing the MA-LSTM model with the MA-LSSVR model from Table 4, the values of MAPE and NMAE predicted by the LSTM model are significantly smaller, indicating that the error percentage of the LSTM model predicted sales are smaller; meanwhile, the NMAE values predicted by the LSTM model are also smaller, indicating that LSTM is more accurate in predicting extreme values in prediction. It can be seen that for this type of product sales forecast, the prediction effect of the LSTM model is significantly better than the prediction effect of the LSSVR model.

We compare the MA-LSTM model and the LSTM model from Tables 3 and Tables 4. In general, the prediction result using the data without seasonal influence is better than the prediction result using the data without seasonal influence. Comparing Weibo Review, Baidu Index, and stock fluctuations independently of seasonal factors to remove the impact of seasonal factors on forecasting results, it can be found that Weibo Review and Baidu Index's data removing seasonal factors affect the forecast accuracy. And the stock data has almost no improvement after removing the influence of seasonal factors.

In this paper, five types of data, real sales, MA-LSTM1, MA-LSSVR1, MA-LSTM8, and MA-LSSVR8, are selected for comparison.as shown in Fig. 4.

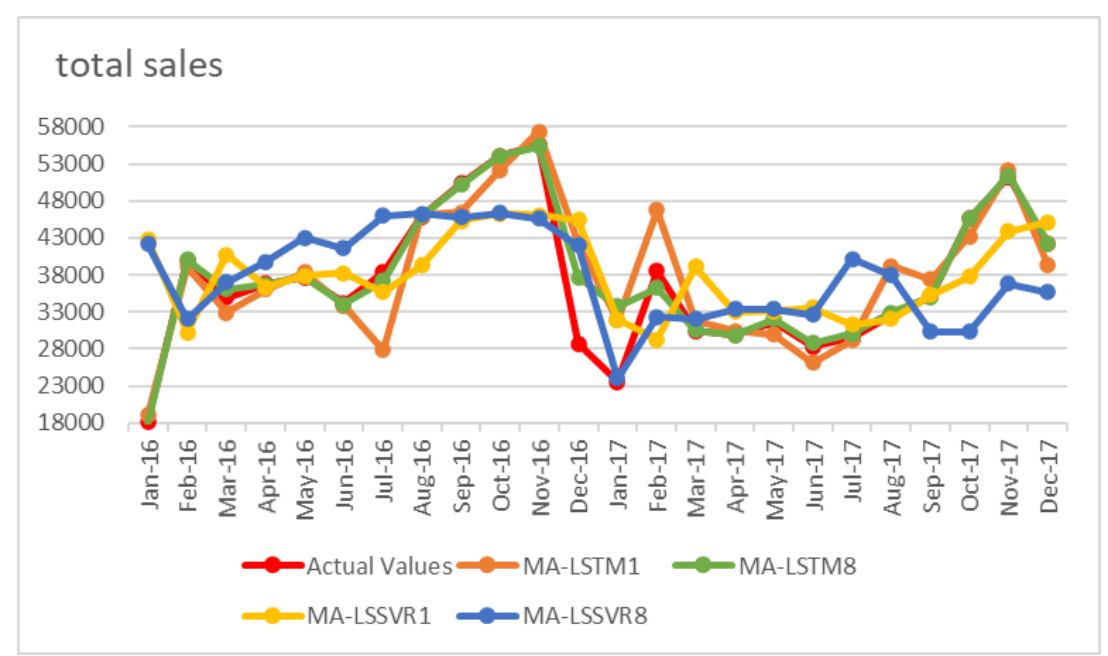

Fig. 4. Actual and predicted deseasonal monthly total sales of LSTM/LSSVR models.

Experimental results were evaluated using the mean percentage error (MAPE). The experimental results show that the prediction error of the cross-value chain data is $42.15 \%$ lower than the prediction result of the single value chain data; the prediction result of the de-seasonal factor data is $15.25 \%$ lower than the prediction result of the data without seasonal factor; the prediction result of LSTM model is 52.15\% lower than that of LSSVR model.

\section{Conclusions}

This research proposes a new algorithm MA-LSTM, which includes a time series prediction model and multivariate regression technology to predict monthly sales. De- 
seasonalization algorithms are used to process different types of data. Numerical results show that using mixed multivariate regression data to predict sales can obtain more accurate prediction results than univariate models. The excellent prediction performance can be concluded as follows. First, using a mix of data that includes portal attention, social media ratings, and stock market value can improve forecast accuracy. Secondly, quantifying the impact of seasonal factors on the attention value of social networking sites and social media evaluation data has significantly improved the accuracy of sales forecasting. The de-seasonal factor of stock data does not greatly improve the accuracy of the sales forecast. It may be because the stock market is more rational, and the correlation between stock market fluctuations and seasonal factors is not great.

In this data set, compared with the prediction of the sales volume of the LSSVR algorithm of PAI and LIU, the MA-LSTM algorithm proposed in this paper is significantly better than the LSSVR algorithm. The reason may be that the LSSVR algorithm has advantages for small sample predictions, but has a poor prediction effect for the 18 products and large data sets of 90 months in this article. Therefore, for data sets with a large amount of data, the MA-LSTM algorithm is significantly better than the LSSVR algorithm.

For future research, since the identification of Weibo keywords has a significant impact on the search results of Weibo and the accuracy of predictions, more systematic techniques for selecting the correct keywords from Weibo maybe future research directions. Another possible direction for future research is to use other social media data, such as the number of product introductions related to video sites, to predict sales. Finally, the collection of geographic information on Weibo may be an important issue for future research to improve Weibo analysis, and shopping preferences of people in different regions may be significantly different.

\section{Acknowledgements}

This work was supported in part by the National Key R\&D Program of China under Grant 2018YFB1702902, and in part by the Shandong Province Colleges and Universities Young Talents Initiation Program under Grant 2019KJN047.

\section{References}

[1] Sa-ngasoongsong A, Bukkapatnam S T S, Kim J, et al.: Multi-step sales forecasting in automotive industry based on structural relationship identification[J]. International Journal of Production Economics. pp. 875-887 (2012)

[2] Wijnhoven F, Plant O.: Sentiment analysis and google trends data for predicting car sales[J] (2017)

[3] Fan Z P, Che Y J, Chen Z Y.: Product sales forecasting using online reviews and historical sales data: A method combining the Bass model and sentiment analysis[J]. Journal of Business Research. pp. 90100 (2017)

[4] Matteo De Felice, Xin Yao.: Notes Short-Term Load Forecasting with Neural Network Ensembles. A Comparative Study[J]. IEEE Computational Intelligence Magazine. pp. 47-56 (2011)

[5] Render B, Stair Jr R M.: Quantitative Analysis for Management, 12e[M]. Pearson Education India. (2016)

[6] Brown R G.: Statistical forecasting for inventory control[M]. McGraw/Hill. (1959)

[7] Trigg D W, Leach A G.: Exponential smoothing with an adaptive response rate[J]. Journal of the Operational Research Society. pp. 53-59 (1967)

[8] Wu S, Rendall J B, Smith M J, et al.: Survey on prediction algorithms in smart homes[J]. IEEE Internet of Things Journal. pp. 636-644 (2017)

[9] Dai H, MacBeth C.: Effects of learning parameters on learning procedure and performance of a BPNN[J]. Neural networks. pp. 1505-1521 (1997)

[10] S. Mukherjee, E. Osuna, and F. Girosi.: Nonlinear prediction of chaotic time series using support vector machines. Workshop Neural Netw. Signal Process. pp. 511-520 (1997) 
[11] Fan M H, Chen M Y, Liao E C.: A deep learning approach for financial market prediction: utilization of Google trends and keywords[J]. Granular Computing. pp. 1-10 (2019)

[12] Yang F, Chen Z, Li J, et al: A novel hybrid stock selection method with stock prediction[J]. Applied Soft Computing. pp. 820-831 (2019)

[13] K. R. Müller, A. J. Smola, G. Rätsch, B. Schölkopf, J. Kohlmorgen, and V. Vapnik.: Predicting time series with support vector machines. Artificial Neural Networks-ICANN. pp. 999-1004 (1997)

[14] V. Vapnik, S. Golowich, and A. Smola.: Support vector method for function approximation, regression estimation and signal processing. Proc. Adv. Neural Inf. Process. Syst. pp. 281-287 (1997)

[15] A. A. Bailey, C. M. Bonifield, and A. Arias.: Social media use by young Latin American consumers: An exploration. J. Retailing Consum. Services, vol. 43. pp. 10-19 (2018)

[16] N. Kim and W. Kim.: Do your social media lead you to make social deal purchases? Consumergenerated social referrals for sales via social commerce. Int. J. Inform. Manage., vol. 39. pp. 38-48 (2018)

[17] H.-L. Chang, Y.-C. Chou, D.-Y. Wu, and S.-C. Wu.: Will firm's marketing efforts on owned social media payoff? A quasi-experimental analysis of tourism products. Decis. Support Syst., vol. 107. pp. 1325 (2018)

[18] S. C. Ludvigson and C. Steindel.: How important is the stock market effect on consumption?. Econ. Policy Rev.-Federal Reserve Bank New York, vol. 5, no. 2. pp. 29-51 (1999)

[19] F. Milani.: Learning about the interdependence between the macroeconomy and the stock market. Int. Rev. Econ. Finance, vol. 49. pp. 223-242 (2017)

[20] Pai P F, Liu C H.: Predicting vehicle sales by sentiment analysis of Twitter data and stock market values[J]. IEEE Access. pp. 57655-57662. (2018)

[21] M. Beladev, L. Rokach, and B. Shapira.: Recommender systems for product bundling. Knowl.Based Syst., vol. 111. pp. 193-206 (2016)

[22] N. Oliveira, P. Cortez, and N. Areal.: The impact of microblogging data for stock market prediction: Using Twitter to predict returns, volatility, trading volume and survey sentiment indices. Expert Syst.

Appl., vol. 73. pp. 125-144 (2017) 